\title{
Fabrication of Large-Area Molybdenum Disulfide Device Arrays Using Graphene/Ti Contacts
}

\author{
Myungwoo Son ${ }^{1,+}$, Jaewon Jang ${ }^{2,+} \mathbb{D}$, Dong Chul $\mathrm{Kim}^{3}{ }^{3}$, Seunghyup Lee ${ }^{3} \mathbb{D}$, Hyo-Soon Shin ${ }^{3}$, Moon-Ho Ham ${ }^{4}$ \\ and Sang-Soo Chee ${ }^{3, *(\mathbb{D}}$
}

1 Artificial Intelligence and Energy Research Center, Korea Photonics Technology Institute (KOPTI), Gwangju 61007, Korea; mwson@kopti.re.kr

2 Gwangju Institute of Science and Technology (GIST), School of Earth Sciences and Environmental Engineering (SESE), Gwangju 61005, Korea; jwjang@gm.gist.ac.kr

3 Nanomaterials and Nanotechnology Center, Korea Institute of Ceramic Engineering and Technology (KICET), Jinju-si 52851, Korea; minamdc@kicet.re.kr (D.C.K.); shbelly@kicet.re.kr (S.L.); hshin@kicet.re.kr (H.-S.S.)

4 Gwangju Institute of Science and Technology (GIST), School of Materials Science and Engineering, Gwangju 61005, Korea; mhham@gist.ac.kr

* Correspondence: sschee@kicet.re.kr

+ These authors contributed equally to this work.

Citation: Son, M.; Jang, J.; Kim, D.C.; Lee, S.; Shin, H.-S.; Ham, M.-H.; Chee, S.-S. Fabrication of Large-Area Molybdenum Disulfide Device Arrays Using Graphene/Ti Contacts. Molecules 2021, 26, 4394. https:// doi.org/10.3390/molecules26154394

Academic Editors: Sung-Hoon Kim and Wan Soo Yun

Received: 15 June 2021

Accepted: 19 July 2021

Published: 21 July 2021

Publisher's Note: MDPI stays neutral with regard to jurisdictional claims in published maps and institutional affiliations.

Copyright: (c) 2021 by the authors. Licensee MDPI, Basel, Switzerland. This article is an open access article distributed under the terms and conditions of the Creative Commons Attribution (CC BY) license (https:// creativecommons.org/licenses/by/ $4.0 /)$.

\begin{abstract}
Two-dimensional (2D) molybdenum disulfide $\left(\mathrm{MoS}_{2}\right)$ is the most mature material in 2D material fields owing to its relatively high mobility and scalability. Such noticeable properties enable it to realize practical electronic and optoelectronic applications. However, contact engineering for large-area $\mathrm{MoS}_{2}$ films has not yet been established, although contact property is directly associated to the device performance. Herein, we introduce graphene-interlayered Ti contacts (graphene/Ti) into large-area $\mathrm{MoS}_{2}$ device arrays using a wet-transfer method. We achieve $\mathrm{MoS}_{2}$ devices with superior electrical and photoelectrical properties using graphene/Ti contacts, with a field-effect mobility of $18.3 \mathrm{~cm}^{2} / \mathrm{V} \cdot \mathrm{s}$, on/off current ratio of $3 \times 10^{7}$, responsivity of $850 \mathrm{~A} / \mathrm{W}$, and detectivity of $2 \times 10^{12}$ Jones. This outstanding performance is attributable to a reduction in the Schottky barrier height of the resultant devices, which arises from the decreased work function of graphene induced by the charge transfer from Ti. Our research offers a direction toward large-scale electronic and optoelectronic applications based on 2D materials.
\end{abstract}

Keywords: $\mathrm{MoS}_{2}$; graphene; interlayer; metal contacts; large-area

\section{Introduction}

Two-dimensional (2D) molybdenum disulfide $\left(\mathrm{MoS}_{2}\right)$ has emerged as a post-silicon material because of its outstanding electrical and optical properties compared with its bulk counterparts [1,2]. In particular, its well-established scaled-up production and tunable bandgap depending on the number of layers make it promising for potential electronic and optoelectronic applications [1-3].

To realize electronic and optoelectronic devices based on $\mathrm{MoS}_{2}$, suitable contact engineering is essential because the Schottky barrier height (SBH) formed between $\mathrm{MoS}_{2}$ and metal contacts is critical for determining the field-effect mobility of the resultant device [4]. Various metal contact approaches have been implemented to improve the device performance over the last decade [4-6]. Das et al. used scandium contacts, an extremely low-work-function metal, to achieve an outstanding mobility $\left(\sim 700 \mathrm{~cm}^{2} / \mathrm{V} \cdot \mathrm{s}\right)[4]$. However, there was a significant difference between the experimental and theoretical SBHs extracted from the Schottky-Mott limit because the metal-induced Fermi level pinning effect prevents a shift in the Fermi level depending on the metal work function $[7,8]$.

Several methods have been developed to overcome metal-induced Fermi level pinning, including interlayer and phase engineering $[9,10]$. In particular, a graphene interlayer can 
suppress metal-induced Fermi level pinning by blocking the interaction between the metal and $\mathrm{MoS}_{2}[9,11]$. Moreover, the interface between graphene and 2D materials is sharp and clean, which leads to efficient charge transfer [12]. Owing to such positive effects, there have been a few reports on graphene-interlayered $\mathrm{MoS}_{2}$ devices $[9,11,13]$, leading to improvements in their electrical properties compared with conventional metal contacts $[9,11]$. However, graphene has a higher work function than $\mathrm{MoS}_{2}[14,15]$, implying that the SBH is even higher than that of conventional low-work-function metal contacts. Furthermore, mechanically exfoliated $\mathrm{MoS}_{2}$ flakes have been commonly utilized, which cannot be applied in large-scale devices because of their limited flake size [9,11]. Therefore, it is highly desirable to develop interlayer engineering that enables practical large-scale electronic and optoelectronic applications while achieving a high performance.

In this study, we developed graphene-interlayered Ti contacts (graphene/Ti contacts) to improve the device performance of large-area $\mathrm{MoS}_{2}$ films grown by chemical vapor deposition (CVD). Ti is a low-work-function metal [16], which can overcome the high work function of graphene because of the efficient Fermi level tunability afforded by metal deposition. To confirm the effects of graphene/Ti contacts, we also fabricate conventional Ti-contacted $\mathrm{MoS}_{2}$ devices and characterize their electrical and photoelectrical properties.

\section{Results and Discussion}

Figure 1a shows the $\mathrm{MoS}_{2}$ film synthesized on a $\mathrm{Si} / \mathrm{SiO}_{2}$ substrate. Overall, the synthesized $\mathrm{MoS}_{2}$ film shows a good uniformity with no noticeable cracks (Figure 1a). High-resolution scanning transmission electron microscopy (STEM) shows a three-fold coordinated lattice structure [17]. A thickness of $0.7 \mathrm{~nm}$ was measured by atomic force microscopy (AFM) analysis, which implies that our $\mathrm{MoS}_{2}$ film is a monolayer (Figure 1b,c) [18]. The Raman spectrum of the $\mathrm{MoS}_{2}$ film shows two main peaks corresponding to $E_{2 \mathrm{~g}}^{1}$ and $A_{1 \mathrm{~g}}$ originating from in-plane and out-of-plane vibrations [18,19], respectively (Figure 1d). From this spectrum, we estimate the peak difference $\left(A_{1 \mathrm{~g}}-E_{2 \mathrm{~g}}^{1}\right)$ and the full-width at halfmaximum (FWHM) of the $E_{2 \mathrm{~g}}^{1}$ peak, which are associated with the number of layers and film quality, respectively $[18,19]$. The peak difference and FWHM were estimated to be approximately 20 and $4.1 \mathrm{~cm}^{-1}$, respectively, which reveal that our $\mathrm{MoS}_{2}$ film is a monolayer and that its quality is comparable to that obtained by mechanical exfoliation $[18,19]$.
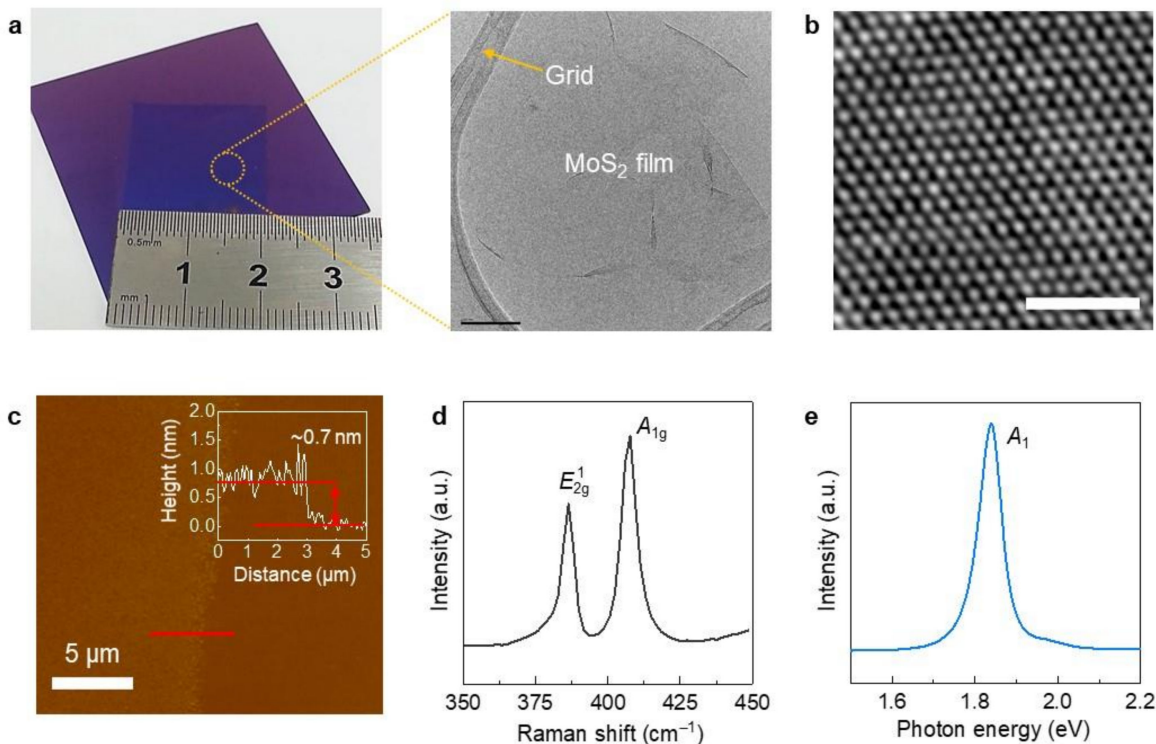

Figure 1. Characterization of $\mathrm{MoS}_{2}$ monolayer film grown by CVD: (a) digital micrograph and TEM images (scale bar denotes $100 \mathrm{~nm}$ ), (b) high-resolution STEM image (scale bar denotes $1 \mathrm{~nm}$ ), (c) AFM image (inset: line profile graph marked by red line), (d) Raman spectrum, and (e) PL spectrum. 
The photoluminescence (PL) spectrum was also obtained to confirm the exciton energy of the $\mathrm{MoS}_{2}$ film (Figure 1e). A strong peak was observed at $1.84 \mathrm{eV}\left(A_{1}\right)$, which further confirms that our $\mathrm{MoS}_{2}$ film is a monolayer [19]. This PL analysis is well matched with the STEM, Raman, and AFM analyses.

Figure 2a shows a schematic illustration of the $\mathrm{MoS}_{2}$ devices with graphene/Ti contacts. The device fabrication step was inspired from Chee et al. [13]. We first synthesized a graphene monolayer film grown by CVD (Figure S1), following a wet transfer on $\mathrm{Si} / \mathrm{SiO}_{2}$ substrate. After that, we fabricated the patterned graphene using a standard photolithography. Once graphene was patterned, we transferred graphene patterns onto CVD-grown $\mathrm{MoS}_{2}$ film using a wet transfer method again. We then patterned the source and drain electrodes on top of the graphene. Finally, we directly deposited 50 nm-thick Ti and then $10 \mathrm{~nm}$-thick Au to prevent the oxidation of Ti. Contrary to methods in previous reports on graphene interlayer-contacted devices, our process enables the realization of large-area $\mathrm{MoS}_{2}$ device arrays (inset in Figure 2a). We explored the electrical properties of the $\mathrm{MoS}_{2}$ devices with $\mathrm{Ti}$ and graphene/Ti contacts to confirm the effect of the graphene interlayer. As shown in Figure $2 \mathrm{~b}, \mathrm{c}$, the $\mathrm{MoS}_{2}$ devices exhibit identical $n$-type behaviors regardless of the type of metal contact. Interestingly, graphene/Ti contacted devices present more lineardominant behavior, and the on-state current level is also significantly enhanced, compared with the Ti contacted devices. Thanks to this improvement, the device performances of the $\mathrm{MoS}_{2}$ device with graphene/Ti contacts are also improved (mobility: $18.3 \mathrm{~cm} / \mathrm{V} \cdot \mathrm{s}$, on/off current ratio: $3.1 \times 10^{7}$ ) relative to the device with Ti contacts (mobility $3.2 \mathrm{~cm}^{2} / \mathrm{V} \cdot \mathrm{s}$, on/off current ratio: $4.5 \times 10^{6}$ ). In general, the enhancement in the field-effect mobility is generally accompanied with a reduction in the on/off current ratio [20], while our device with graphene/Ti contacts presents an identical off-state current level, compared with conventional Ti contacted devices.

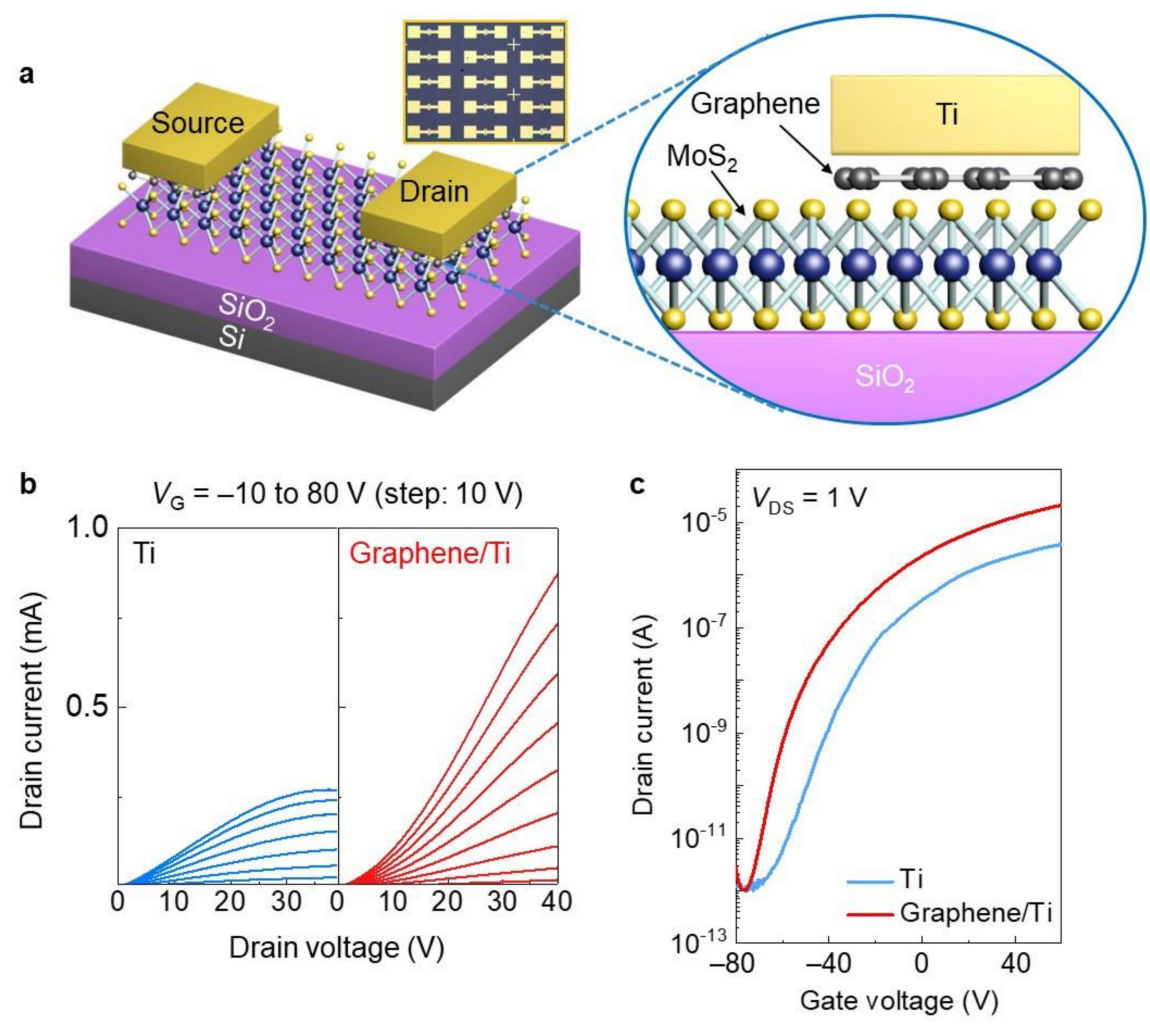

Figure 2. (a) Schematic illustration of graphene/Ti-contacted $\mathrm{MoS}_{2}$ device (inset: optical microscopy image of $\mathrm{MoS}_{2}$ device arrays with graphene/Ti contacts). (b) Output and (c) transfer characterizations of $\mathrm{MoS}_{2}$ devices with $\mathrm{Ti}$ and graphene/Ti contacts. 
This outstanding improvement is attributed to the reduced SBHs of the resultant devices using the graphene/ $\mathrm{Ti}$ contacts, leading to efficient charge transfer from the graphene/Ti contacts to the $\mathrm{MoS}_{2}$ channel [13,21].

To prove our hypothesis, we quantitatively estimate the SBHs using a modified Richardson plot inspired by Tataroğlu et al. [22]:

$$
\ln \left(\frac{I_{0}}{T^{2}}\right)-\left(\frac{q^{2} \sigma^{2}}{2 k^{2} T^{2}}\right)=\ln \left(A A^{*}\right)-\frac{q \varphi_{B}}{k T}
$$

where $I_{0}$ is the saturation current level, $T$ is the temperature $(\mathrm{K}), q$ is the electronic charge, $k$ is the Boltzmann constant, $\sigma$ is the standard deviation of the Gaussian function of the $\mathrm{SBH}, A$ is the device area, $A^{*}$ is the Richardson constant, and $\varphi_{\mathrm{B}}$ is the barrier height. The Richardson constant is strongly related to the effective mass of the material and can be defined as $A^{*}=4 \pi q m^{*} k^{2} / h^{3}$, where $m^{*}$ is the effective mass of $\operatorname{MoS}_{2}$ and $h$ is Planck's constant $[22,23]$. From these equations, we plotted $\ln \left(I_{0} / T^{2}\right)-\left[\left(q^{2} \sigma^{2}\right) /\left(2 k^{2} T^{2}\right)\right]$ of the $\mathrm{MoS}_{2}$ devices with Ti and graphene/Ti contacts as a function of $q /(k T)$ (Figure S2). Using these graphs, the SBHs for each $\mathrm{MoS}_{2}$ device can be extracted as a function of the gate voltage by linear fitting under flat-band gate voltage conditions (Figure 3a), which corresponds to a point of deviation from the linear fit $[4,13]$. The extracted SBHs of the devices with $\mathrm{Ti}$ and graphene/Ti contacts were 0.40 and $0.31 \mathrm{eV}$, respectively. The reduced SBH with the graphene/Ti contacts was accompanied by a reduction in the contact resistance $\left(R_{\mathrm{C}}\right)$ with respect to the device with the Ti contacts, which is a result of an improvement in the electrical properties (Figures $2 \mathrm{c}$ and $3 \mathrm{~b}$ ).
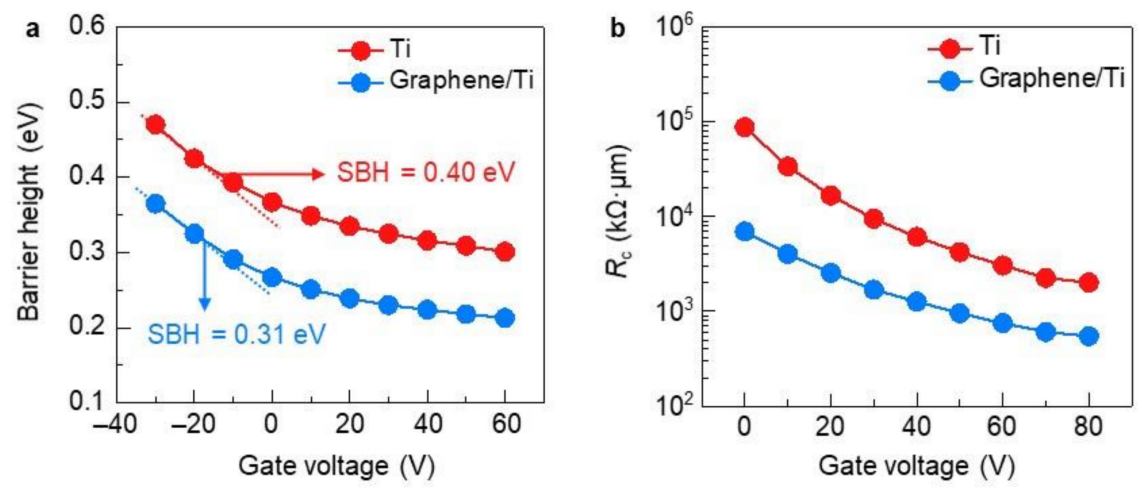

c

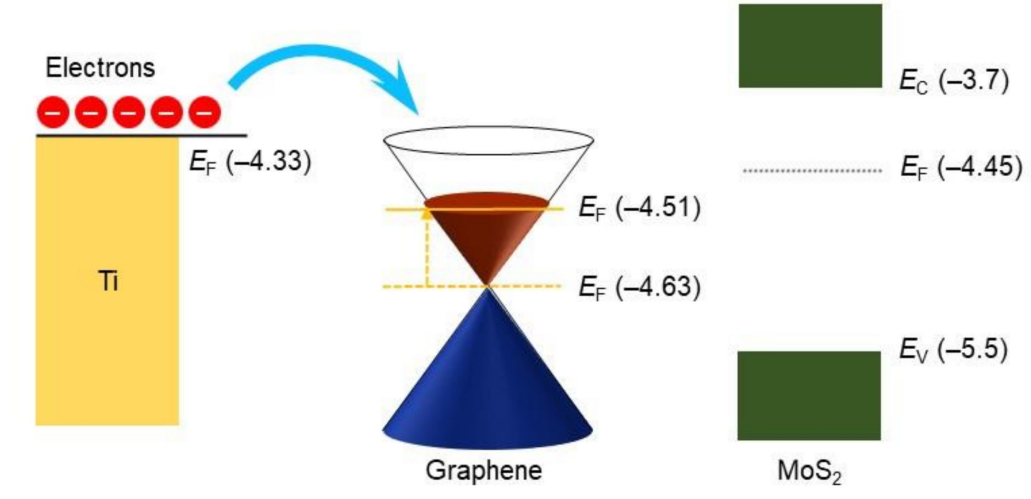

Figure 3. (a) Barrier height and (b) contact resistance $\left(R_{\mathrm{c}}\right)$ of $\mathrm{MoS}_{2}$ devices with Ti and graphene/Ti contacts. Barrier heights were obtained from the data in Figure S2. (c) Band alignment of $\mathrm{MoS}_{2}$ monolayer contacted with graphene/Ti, where the Fermi levels $\left(E_{\mathrm{F}}\right)$ of graphene and $\mathrm{MoS}_{2}$ were extracted from the data in Figure S3. 
This result correlates with output and transfer characterizations (Figure $2 b, c)$. Wang et al. also demonstrated that the use of an interlayer can enhance the electrical properties of $\mathrm{MoS}_{2}$ devices by reducing the SBH [24].

To unveil why the SBH is reduced with the use of the graphene/Ti contacts in detail, ultraviolet photoelectron spectroscopy (UPS) measurements were carried out (Figure $3 c$ and Figure S3). The work function of pristine graphene is approximately $4.63 \mathrm{eV}$, which is higher than that of $\mathrm{MoS}_{2}(4.45 \mathrm{eV})$. This means that a higher SBH would be formed between pristine graphene and $\mathrm{MoS}_{2}$. In contrast, the work function of graphene on a Ti film appears to be $4.51 \mathrm{eV}$, which implies that Ti induces an $n$-type doping effect on graphene [13,25], and consequently, the SBH can be lowered when graphene/Ti is in contact with $\mathrm{MoS}_{2}$ (Figure 3a,c). Considering that the lower work function metal is utilized rather than Ti, the SBH would be further reduced, and the electrical properties would also be enhanced. This result can be also supported by a transfer curve of an only graphene-contacted device, but not an interlayer contacted one (Figure S4). Its device performance is also improved (mobility: $7.8 \mathrm{~cm}^{2} / \mathrm{Vs}$ ) compared with Ti contacted devices, but the contact effect is weaker than the graphene/Ti contacted device. This suggests that the coupling with graphene and low work function metals (Ti), is significantly beneficial to the $\mathrm{MoS}_{2}$ device performance.

Next, we characterized the photoelectrical properties of the $\mathrm{MoS}_{2}$ devices depending on the metal contacts under light illumination at $470 \mathrm{~nm}$ (Figure 4). Both the Ti- and graphene/Ti-contacted devices showed increased photocurrent levels under illumination, but the increase was larger for the graphene/Ti-contacted device. This is attributed to the boosted mobility resulting from the reduced SBH [13]. To quantify the photoelectrical performance, we estimated the power-dependent responsivity $(R)$, defined as $R=I_{\mathrm{ph}} /(A P)$, where $I_{\mathrm{ph}}$ is the photocurrent, $A$ is the device area, and $P$ is the light power density (Figure 4a).
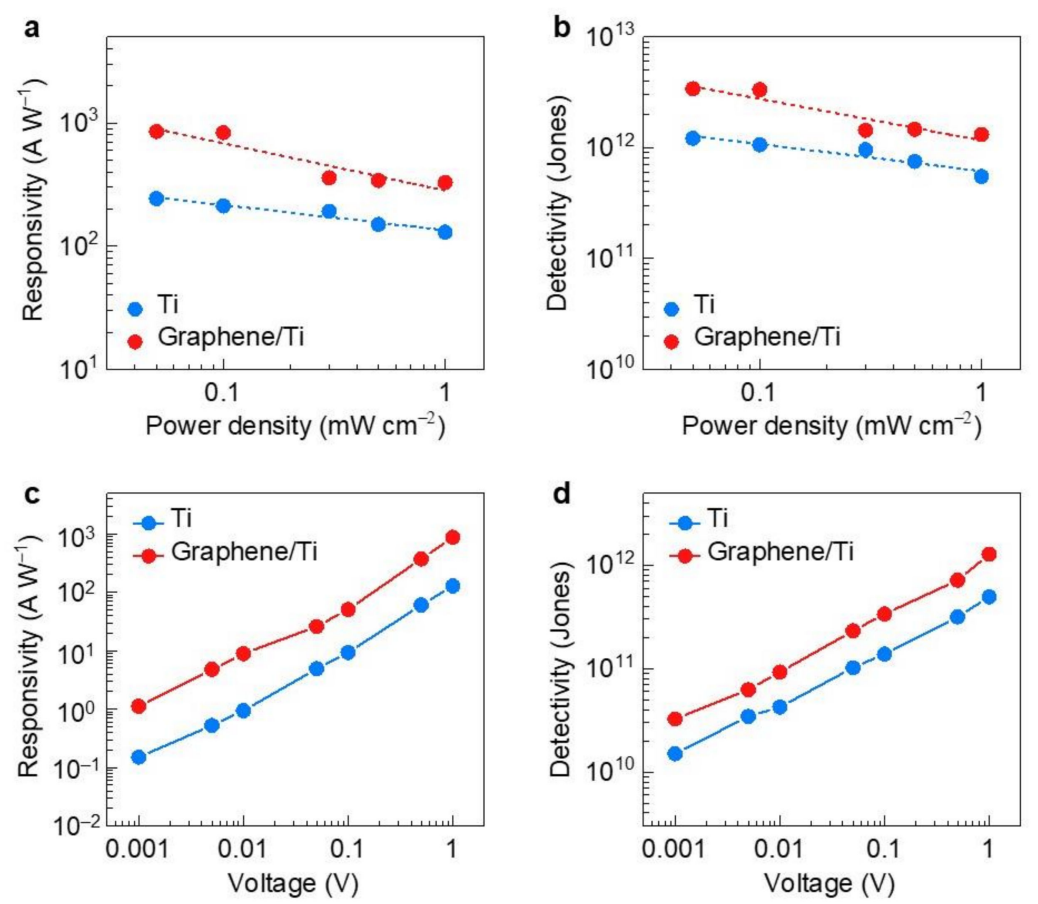

Figure 4. (a) Responsivities and (b) detectivities of $\mathrm{MoS}_{2}$ devices with Ti and graphene/Ti contacts under an applied bias of $1 \mathrm{~V}$ and $\mathrm{V}_{\mathrm{G}}=0 \mathrm{~V}$ and light illumination at $470 \mathrm{~nm}$ as a function of light power density. (c) Responsivities and (d) detectivities of $\mathrm{MoS}_{2}$ devices with Ti and graphene/Ti contacts under light illumination at $470 \mathrm{~nm}$ as a function of an applied bias, under $\mathrm{V}_{\mathrm{G}}=0 \mathrm{~V}$. All graphs were acquired from the data in Figure S5. 
For both devices, the responsivity decreases linearly with increasing power density. This is associated with an increase in scattering events between photogenerated carriers due to the high-power density $[13,26]$, and a behavior that has been commonly demonstrated in TMDC photodetector devices. We note that the maximal responsivity of the device with graphene/Ti contacts is approximately $850 \mathrm{~A} / \mathrm{W}$, which is significantly higher (by a factor of 3.5) than that of the Ti-contacted device. This value is also considerably higher than previously reported values from studies on the performance of TMDC photodetectors [27,28].

The detectivity $\left(D^{*}\right)$ is another figure of merit for the sensitivity of photodetectors, which can be expressed as $D^{*}=R A^{1 / 2} /\left(2 q I_{\text {dark }}\right)^{1 / 2}$, where $R$ is the responsivity, $A$ is the device area, $q$ is the electronic charge, and $I_{\text {dark }}$ is the dark current. A detectivity of $\sim 2 \times 10^{12}$ Jones is achieved for the device with graphene/Ti contacts, which is higher than that of the Ti-contacted devices (Figure $4 \mathrm{~b}$ ). Lastly, we characterized the responsivities and detectivities as a function of the applied bias (Figure $4 c, d$ ). With an increase in the applied bias, the responsivities and detectivities increased linearly for both contacted devices, but the values of graphene/ Ti contacted devices were higher, with respect to Ti contacted one. This means that the outstanding photoelectrical performance of the graphene/Ti-contacted device at even an extremely low bias can be attributed to an improved mobility and low recombination rate between photogenerated carriers [29].

\section{Materials and Methods}

\subsection{Synthesis of $\mathrm{MoS}_{2}$ and Graphene Monolayer Films}

The $\mathrm{MoS}_{2}$ film was synthesized by CVD using both powders, $\mathrm{MoO}_{3}$ (99.999\%, Materion Advanced Chemicals, Seoul, Korea) and sulfur (99.9\% Sigma Aldrich, Saint Louis, MO, USA). Each powder was loaded into two separate alumina crucibles; the $\mathrm{MoO}_{3}$ crucible was positioned at the center of a quartz tube, and the sulfur crucible was placed upstream of the $\mathrm{MoO}_{3}$ crucible. A Si $/ \mathrm{SiO}_{2}$ substrate was placed downstream of the $\mathrm{MoO}_{3}$ crucible. The tube furnace was heated to $650{ }^{\circ} \mathrm{C}$ and then kept for 40 min under $50 \mathrm{sccm}$ of Ar carrier gas at 300 mTorr. After that, the tube furnace was naturally cooled to room temperature with $200 \mathrm{sccm}$ of Ar gas. A graphene film was synthesized on a Cu catalyst (Alfa Aesar, Ward Hill, MA, USA) using CVD by a process similar to the $\mathrm{MoS}_{2}$ synthesis. The detailed synthesis procedure is described in our previous report [30].

\subsection{Fabrication of $\mathrm{MoS}_{2}$ Devices with Ti and Graphene/Ti Contacts}

$\mathrm{MoS}_{2}$ devices were fabricated using a standard photolithography method. First, $\mathrm{MoS}_{2}$ films were synthesized on a heavily $p$-doped $\mathrm{Si}$ substrate with a $300 \mathrm{~nm}$-thick $\mathrm{SiO}_{2}$ layer, which acted as the bottom gate and dielectric layer, respectively. To prepare the patterned graphene, we used standard photolithography and an oxygen plasma etching system. Once graphene was patterned, the graphene electrodes were transferred onto the $\mathrm{MoS}_{2}$ film using a wet-transfer method, where a buffered oxide etch solution was used to etch the $\mathrm{SiO}_{2}$ layer. Then, secondary photolithography was performed on the graphene electrodes, followed by metal deposition ( $50 \mathrm{~nm}$-thick Ti and then $10 \mathrm{~nm}$-thick $\mathrm{Au}$ ). Channel definition was conducted with a length of $14 \mu \mathrm{m}$ and width of $40 \mu \mathrm{m}$ using a third photolithography step, and then an unprotected $\mathrm{MoS}_{2}$ area was etched using Ar plasma treatment. For comparison, a $\mathrm{MoS}_{2}$ device with Ti contacts was prepared by identical processes except for preparation of the graphene electrodes.

\subsection{Characterization}

We performed optical microscopy (BX51, Olympus, Seoul, Korea), AFM (XE-100, Park Systems, Suwon, Korea), and Raman and PL spectroscopy (LabRAM HR Evolution, Horiba Jovin-Yvon, Kyoto, Japan) measurements to investigate the physical properties of the $\mathrm{MoS}_{2}$ films grown by CVD. To explore the work functions of the $\mathrm{MoS}_{2}$ and graphene films, UPS (Riken Keiki instrument, AC-2, Tokyo, Japan) measurements were carried out. To characterize the electrical and photoelectrical properties of the resultant devices, we used a 
semiconducting parameter analyzer (E5270B, Agilent Technologies, Santa Clara, CA, USA) under vacuum ( $\left.10^{-5} \mathrm{mbar}\right)$ at $80-300 \mathrm{~K}$ and a visible light lamp $(470 \mathrm{~nm})$.

\section{Conclusions}

In conclusion, we fabricated CVD-grown $\mathrm{MoS}_{2}$ device arrays with graphene/Ti contacts via a wet-transfer method. Using the graphene/Ti contacts, we achieved remarkable electrical and photoelectrical performances compared with the Ti-contacted devices, including a field-effect mobility of $18.3 \mathrm{~cm}^{2} / \mathrm{V} \cdot \mathrm{s}$, on/off current ratio of $3.1 \times 10^{7}$, responsivity of $850 \mathrm{~A} / \mathrm{W}$, and detectivity of $2 \times 10^{12}$ Jones. Such enhancements can be explained by the reduced SBHs coming from a decrease in the work function of the graphene interlayer induced by electron charge transfer from the low-work-function Ti metal. This interaction is more beneficial to boost device performances, compared with only graphene-contacted devices. Our contact engineering enables easy expansion of the doping range of graphene using various metal contacts, and directly combines high-k dielectric layers [31], achieving the high-performance 2D devices. This also facilitates realization of the 2D TMDC-based photodiode [32], thanks to the Fermi level de-pinning effect. Therefore, our findings provide insight for realizing practical large-scale electronic and optoelectronic applications based on 2D TMDCs.

Supplementary Materials: Figure S1: Characterization of graphene film, Figure S2: $\ln \left(I_{0} / T^{2}\right)-$ $\left(q^{2} \sigma^{2} / 2 K^{2} T^{2}\right)$ vs. $q / K T$ plots depending on contact types, Figure S3: UPS results of $\mathrm{MoS}_{2}$, Ti and graphene/Ti films, Figure S4: transfer characterizations of $\mathrm{MoS}_{2}$ devices with Ti, graphene, and graphene/Ti contacts, Figure S5: power-dependent $I-V$ curves of $\mathrm{MoS}_{2}$ devices with Ti and graphene/Ti contacts.

Author Contributions: Graphene synthesis, writing —original draft preparation, M.S. and J.J.; writing - original draft preparation; S.L. and H.-S.S.; $\mathrm{MoS}_{2}$ synthesis, device fabrication and characterization, writing —original draft preparation, D.C.K., M.-H.H. and S.-S.C. All authors have read and agreed to the published version of the manuscript.

Funding: This work was supported by the National Research Foundation of Korea (NRF) grant funded by the Korea government (MSIT) (No. 2021R1F1A1059833), "Basic Science Research program" funded by the Korea Institute of Ceramic Engineering and Technology, Republic of Korea (No. KPB21003), Ministry of Trade, Industry and Energy (MOTIE) and Korea Evaluation Institute of Industrial Technology (No. 20003891), the National Research Foundation (NRF) of Korea funded by the Ministry of Science \& ICT (No. 2020R1F1A1069342), and GIST Research Project grant funded by the GIST in 2021.

Institutional Review Board Statement: Not applicable.

Informed Consent Statement: Not applicable.

Data Availability Statement: The data presented in this study are available on request from the corresponding author.

Conflicts of Interest: The authors declare no conflict of interest.

Sample Availability: Samples of the compounds are not available from the authors.

\section{References}

1. Butler, S.Z.; Hollen, S.M.; Cao, L.; Cui, Y.; Gupta, J.A.; Gutiérrez, H.R.; Heinz, T.F.; Hong, S.S.; Huang, J.; Ismach, A.F.; et al. Progress, Challenges, and Opportunities in Two-Dimensional Materials Beyond Graphene. ACS Nano 2013, 7, 2898-2926. [CrossRef]

2. Wang, Q.H.; Kalantar-Zadeh, K.; Kis, A.; Coleman, J.N.; Strano, M.S. Electronics and Optoelectronics of Two-Dimensional Transition Metal Dichalcogenides. Nat. Nanotechnol. 2012, 7, 699-712. [CrossRef] [PubMed]

3. Yu, H.; Liao, M.; Zhao, W.; Liu, G.; Zhou, X.J.; Wei, Z.; Xu, X.; Liu, K.; Hu, Z.; Deng, K.; et al. Wafer-Scale Growth and Transfer of Highly-Oriented Monolayer $\mathrm{MoS}_{2}$ Continuous Films. ACS Nano 2017, 11, 12001-12007. [CrossRef] [PubMed]

4. Das, S.; Chen, H.-Y.; Penumatcha, A.V.; Appenzeller, J. High Performance Multilayer $\mathrm{MoS}_{2}$ Transistors with Scandium Contacts. Nano Lett. 2013, 13, 100-105. [CrossRef] [PubMed] 
5. Kang, J.; Liu, W.; Banerjee, K. High-Performance $\mathrm{MoS}_{2}$ Transistors with Low-Resistance Molybdenum Contacts. Appl. Phys. Lett. 2014, 104, 093106. [CrossRef]

6. Abraham, M.; Mohney, S.E. Annealed Ag Contacts to MoS 2 Field-Effect Transistors. J. Appl. Phys. 2017, 122, 115306. [CrossRef]

7. Bampoulis, P.; van Bremen, R.; Yao, Q.; Poelsema, B.; Zandvliet, H.J.W.; Sotthewes, K. Defect Dominated Charge Transport and Fermi Level Pinning in $\mathrm{MoS}_{2}$ /Metal Contacts. ACS Appl. Mater. Interfaces 2017, 9, 19278-19286. [CrossRef] [PubMed]

8. Kim, C.; Moon, I.; Lee, D.; Choi, M.S.; Ahmed, F.; Nam, S.; Cho, Y.; Shin, H.-J.; Park, S.; Yoo, W.J. Fermi Level Pinning at Electrical Metal Contacts of Monolayer Molybdenum Dichalcogenides. ACS Nano 2017, 11, 1588-1596. [CrossRef] [PubMed]

9. Leong, W.S.; Luo, X.; Li, Y.; Khoo, K.H.; Quek, S.Y.; Thong, J.T.L. Low Resistance Metal Contacts to MoS 2 Devices with Nickel-Etched-Graphene Electrodes. ACS Nano 2015, 9, 869-877. [CrossRef]

10. Zheng, J.; Yan, X.; Lu, Z.; Qiu, H.; Xu, G.; Zhou, X.; Wang, P.; Pan, X.; Liu, K.; Jiao, L. High-Mobility Multilayered MoS 2 Flakes with Low Contact Resistance Grown by Chemical Vapor Deposition. Adv. Mater. 2017, 29, 1604540. [CrossRef]

11. Du, Y.; Yang, L.; Zhang, J.; Liu, H.; Majumdar, K.; Kirsch, P.D.; Ye, P.D. $\mathrm{MoS}_{2}$ Field-Effect Transistors With Graphene/Metal Heterocontacts. IEEE Electron. Device Lett. 2014, 35, 599-601.

12. Liu, Y.; Wu, H.; Cheng, H.-C.; Yang, S.; Zhu, E.; He, Q.; Ding, M.; Li, D.; Guo, J.; Weiss, N.O.; et al. Toward Barrier Free Contact to Molybdenum Disulfide Using Graphene Electrodes. Nano Lett. 2015, 15, 3030-3034. [CrossRef]

13. Chee, S.-S.; Seo, D.; Kim, H.; Jang, H.; Lee, S.; Moon, S.P.; Lee, K.H.; Kim, S.W.; Choi, H.; Ham, M.-H. Lowering the Schottky Barrier Height by Graphene/Ag Electrodes for High-Mobility $\mathrm{MoS}_{2}$ Field-Effect Transistors. Adv. Mater. 2019, 31 , 1804422. [CrossRef]

14. Lee, Y.T.; Choi, K.; Lee, H.S.; Min, S.-W.; Jeon, P.J.; Hwang, D.K.; Choi, H.J.; Im, S. Graphene Versus Ohmic Metal as Source-Drain Electrode for $\mathrm{MoS}_{2}$ Nanosheet Transistor Channel. Small 2014, 10, 2356-2361. [CrossRef] [PubMed]

15. Maiti, R.; Haldar, S.; Majumdar, D.; Singha, A.; Ray, S.K. Hybrid Opto-Chemical Doping in Ag Nanoparticle-Decorated Monolayer Graphene Grown by Chemical Vapor Deposition Probed by Raman Spectroscopy. Nanotechnology 2017, 28, 075707. [CrossRef] [PubMed]

16. Yuan, H.; Cheng, G.; You, L.; Li, H.; Zhu, H.; Li, W.; Kopanski, J.J.; Obeng, Y.S.; Hight Walker, A.R.; Gundlach, D.J.; et al. Influence of Metal-MoS 2 Interface on $\mathrm{MoS}_{2}$ Transistor Performance: Comparison of Ag and Ti Contacts. ACS Appl. Mater. Interfaces 2015, 7, 1180-1187. [CrossRef]

17. Yan, A.; Chen, W.; Ophus, C.; Ciston, J.; Lin, Y.; Persson, K.; Zettl, A. Identifying Different Stacking Sequences in Few-Layer CVD-Grown $\mathrm{MoS}_{2}$ by Low-Energy Atomic-Resolution Scanning Transmission Electron Microscopy. Phys. Rev. B 2016, $93,041420$. [CrossRef]

18. Jeon, J.; Jang, S.K.; Jeon, S.M.; Yoo, G.; Jang, Y.H.; Park, J.-H.; Lee, S. Layer-Controlled CVD Growth of Large-Area TwoDimensional $\mathrm{MoS}_{2}$ Films. Nanoscale 2015, 7, 1688-1695. [CrossRef] [PubMed]

19. Zhang, J.; Yu, H.; Chen, W.; Tian, X.; Liu, D.; Cheng, M.; Xie, G.; Yang, W.; Yang, R.; Bai, X.; et al. Scalable Growth of High-Quality Polycrystalline $\mathrm{MoS}_{2}$ Monolayers on $\mathrm{SiO}_{2}$ with Tunable Grain Sizes. ACS Nano 2014, 8, 6024-6030. [CrossRef]

20. Du, Y.; Liu, H.; Neal, A.T.; Si, M.; Ye, P.D. Molecular Doping of Multilayer $\mathrm{MoS}_{2}$ Field-Effect Transistors: Reduction in Sheet and Contact Resistances. IEEE Electron. Device Lett. 2013, 34, 1328-1330. [CrossRef]

21. Liu, W.; Kang, J.; Sarkar, D.; Khatami, Y.; Jena, D.; Banerjee, K. Role of Metal Contacts in Designing High-Performance Monolayer n-Type WSe 2 Field Effect Transistors. Nano Lett. 2013, 13, 1983-1990. [CrossRef] [PubMed]

22. Tataroğlu, A.; Pür, F.Z. The Richardson Constant and Barrier Inhomogeneity at Au/Si $3 \mathrm{~N}_{4} / \mathrm{n}-\mathrm{Si}$ (MIS) Schottky Diodes. Phys. Scr. 2013, 88, 015801. [CrossRef]

23. Wang, L.; Kutana, A.; Yakobson, B.I. Many-Body and Spin-Orbit Effects on Direct-Indirect Band Gap Transition of Strained Monolayer $\mathrm{MoS}_{2}$ and $\mathrm{WS}_{2}$. Ann. Phys. 2014, 526, L7-L12. [CrossRef]

24. Wang, J.; Yao, Q.; Huang, C.-W.; Zou, X.; Liao, L.; Chen, S.; Fan, Z.; Zhang, K.; Wu, W.; Xiao, X.; et al. High Mobility MoS 2 Transistor with Low Schottky Barrier Contact by Using Atomic Thick H-BN as a Tunneling Layer. Adv. Mater. 2016, 28, 8302-8308. [CrossRef] [PubMed]

25. Lee, J.-K.; Sung, H.; Jang, M.S.; Yoon, H.; Choi, M. Reliable Doping and Carrier Concentration Control in Graphene by AerosolDerived Metal Nanoparticles. J. Mater. Chem. C 2015, 3, 8294-8299. [CrossRef]

26. Chee, S.-S.; Lee, J.-H.; Lee, K.; Ham, M.-H. Defect-Assisted Contact Property Enhancement in a Molybdenum Disulfide Monolayer. ACS Appl. Mater. Interfaces 2020, 12, 4129-4134. [CrossRef] [PubMed]

27. Wang, X.; Wang, P.; Wang, J.; Hu, W.; Zhou, X.; Guo, N.; Huang, H.; Sun, S.; Shen, H.; Lin, T.; et al. Ultrasensitive and Broadband $\mathrm{MoS}_{2}$ Photodetector Driven by Ferroelectrics. Adv. Mater. 2015, 27, 6575-6581. [CrossRef] [PubMed]

28. Chee, S.-S.; Oh, C.; Son, M.; Son, G.-C.; Jang, H.; Yoo, T.J.; Lee, S.; Lee, W.; Hwang, J.Y.; Choi, H.; et al. Sulfur Vacancy-Induced Reversible Doping of Transition Metal Disulfides via Hydrazine Treatment. Nanoscale 2017, 9, 9333-9339. [CrossRef]

29. Khalil, H.M.W.; Khan, M.F.; Eom, J.; Noh, H. Highly Stable and Tunable Chemical Doping of Multilayer WS 2 Field Effect Transistor: Reduction in Contact Resistance. ACS Appl. Mater. Interfaces 2015, 7, 23589-23596. [CrossRef]

30. Son, M.; Kim, H.; Jang, J.; Kim, S.-Y.; Ki, H.C.; Lee, B.H.; Kim, I.S.; Ham, M.-H. Low-Power Complementary Logic Circuit Using Polymer-Electrolyte-Gated Graphene Switching Devices. ACS Appl. Mater. Interfaces 2019, 11, 47247-47252. [CrossRef] 
31. Noumbé, U.N.; Gréboval, C.; Livache, C.; Brule, T.; Doudin, B.; Ouerghi, A.; Lhuillier, E.; Dayen, J.-F. Ionic Glass-Gated 2D Material-Based Phototransistor: $\mathrm{MoSe}_{2}$ over $\mathrm{LaF}_{3}$ as Case Study. Adv. Funct. Mater. 2019, 29, 1902723. [CrossRef]

32. Noumbé, U.N.; Gréboval, C.; Livache, C.; Chu, A.; Majjad, H.; Parra López, L.E.; Mouafo, L.D.N.; Doudin, B.; Berciaud, S.; Chaste, J.; et al. Reconfigurable 2D/OD p-n Graphene/HgTe Nanocrystal Heterostructure for Infrared Detection. ACS Nano 2020, 14, 4567-4576. [CrossRef] [PubMed] 